\title{
Tinnitus Sound Therapy Trial Shows Effectiveness for Those with Tinnitus
}

DOI: $10.3766 /$ jaaa. 18027

\author{
Richard S. Tyler* \\ Ann Perreau $\dagger$ \\ Thomas Powerst \\ Alexandra Watts $\dagger$ \\ Rachael Owen* \\ Helena Ji* \\ Patricia C. Mancini*§
}

\begin{abstract}
Background: It is well accepted among clinicians that maskers and hearing aids combined with counseling are generally helpful to tinnitus patients, but there are few controlled studies exploring the efficacy of maskers alone to decrease the prominence of tinnitus.
\end{abstract}

Purpose: We investigated the benefit of maskers for patients with chronic, bothersome tinnitus.

Research Design: Crossover single-participant design, where each participant served as their own control.

Study Sample: 18 adults with subjective, nonpulsatile, sensorineural tinnitus.

Intervention: Participants participated in two six-week trials: one with sound therapy and one without. No counseling was provided in either group. Masking devices were fit with sounds intended to reduce the tinnitus prominence.

Data Collection and Analysis: Participants rated tinnitus loudness, tinnitus annoyance, and acceptability of the background sounds using a numeric 0-100 interval scale and completed the Tinnitus Primary Functions Questionnaire (TPFQ).

Results: Three participants dropped out. On the total score of the TPFQ, 5 of 15 remaining participants (33\%) showed a benefit. Using a derived score based on functions showing a handicap before the study, maskers benefit was observed in the areas of sleep (five of nine), hearing (three of eight), thoughts and emotions (three of four), and concentration (four of eight). The TPFQ and annoyance data complemented each other well.

Conclusions: This study demonstrates the benefit of partial masking, encouraging patients to seek help from audiologists interested in providing support for tinnitus patients.

Key Words: partial masking, sound therapy, tinnitus, tinnitus maskers, Tinnitus Primary Functions Questionnaire

Abbreviation: TPFQ $=$ Tinnitus Primary Functions Questionnaire

*Department of Otolaryngology-Head and Neck Surgery, University of lowa, Iowa City, IA; †Department of Communication Sciences and Disorders, Augustana College, Rock Island, IL; + Sivantos, Inc., Piscataway, NJ; §Department of Speech-Language Pathology and Audiology, Universidade Federal de Minas Gerais, Belo Horizonte, Brazil

Corresponding author: Richard S. Tyler, Department of Otolaryngology-Head and Neck Surgery, University of lowa, Iowa City, IA 52242; Email: rich-tyler@uiowa.edu

Sivantos, Inc. provided financial support for the study and Conselho Nacional de Desenvolvimento Cientifico e Tecnologico (CNPq), Brazil, provided a scholarship for Dr. Mancini. This research was also funded by a Summer Research stipend and a Student-Faculty Academic Partnership grant from Augustana College awarded to Ann Perreau and WVIK, who assisted with marketing the study at Augustana College. 


\section{INTRODUCTION}

$\mathrm{T}$ here is currently no cure for tinnitus, but there are many treatment options for those whose quality of life is adversely affected by tinnitus. These include counseling, hearing aids, and tinnitus maskers. Counseling plays an important role on tinnitus treatment. Many counseling therapies have been developed and adapted to treat tinnitus patients (e.g., cognitive behavior therapy: Sweetow, 2000; Henry and Wilson, 2001; progressive audiologic tinnitus management: Henry et al, 2005; tinnitus retraining therapy: Jastreboff, 2000; person-centered therapy: Mohr and Hedelund, 2006; and tinnitus activities treatment: Tyler et al, 2006a; 2007a).

The use of hearing aids has long been recommended for tinnitus relief (Vernon and Schleuning, 1978; Coles, 1987; Hazell et al, 1985). Many studies reported a decrease in the prominence of tinnitus in up to $85 \%$ of patients with sensorineural hearing loss after hearing aid fitting (Hazell et al, 1985; Surr et al, 1985; Tyler and Bentler, 1987; Sandlin and Olsson, 1999; Henry et al, 2015). Many patients report tinnitus has helped when they receive hearing aids (Moffat et al, 2009; Schaette et al, 2010; Kochkin et al, 2011).

Maskers also have been used for tinnitus patients. Vernon (1977) was instrumental in developing the first wearable device to totally or partially mask tinnitus in patients without hearing loss. Tyler and Bentler (1987) proposed specific strategies for fitting tinnitus maskers. They noted that large differences exist across patients on their preference for types of spectral characteristics of maskers. They also recommended that tinnitus maskers should be designed to provide a partial masking, at a lower level to interfere less with speech and to decrease the chance of exacerbating the tinnitus.

Many studies have attempted to evaluate the efficacy of maskers and have reported tinnitus relief in up to $83 \%$ of the individuals using total or partial masking delivered by sound therapy devices (Vernon and Schleuning, 1978; Hazell et al, 1985; Surr et al, 1985; Jastreboff and Hazell, 1993; Vernon and Meikle, 2000; Bauer et al, 2017; Henry et al, 2017). The American Academy of Otolaryngology-Head and Neck Surgery Clinical Practice Guidelines did recommend that sound therapy be offered to patients with bothersome tinnitus, acknowledging that additional data were needed (Tunkel et al, 2014; see also; Moffat et al, 2009; Hobson et al, 2010; Schaette et al, 2010). More specifically, the designs, acoustic stimuli, and outcome measures used to evaluate effectiveness of sound therapies differ significantly in previous studies (Moffat et al, 2009; Hobson et al, 2010; Schaette et al, 2010). Furthermore, as found by Hobson et al (2010), previous studies of sound therapy have been of low quality or have not shown a significant benefit using the masker alone. Despite this lack of evidence, many clinicians provide tinnitus maskers and some patients continue to report relief and purchase maskers (Tyler, 2012).

It is well accepted among clinicians that maskers and hearing aids combined with counseling are generally helpful to tinnitus patients, but there are few controlled studies exploring the efficacy of maskers alone to decrease the prominence of tinnitus. In this study, we used a single-participant design (Byiers et al, 2012) to evaluate the benefit of partial masking for patients with chronic, bothersome tinnitus. We have argued that individual differences can be large in tinnitus patients, and we do not expect to help everyone; thus, withinsubject analyses are more appropriate than grouping data (Tyler et al, 2007b). In this crossover trial, Group A used the sound therapy for the first six weeks, then the no-sound therapy for the next six weeks. Group B received the no-sound therapy for the first six weeks, then the sound therapy for next six weeks.

We excluded the provision of hearing aid amplification and counseling. Not providing counseling is, of course, not appropriate for clinical work, but excluding these variables was important for the design of the study and to provide clinicians with definitive evidence regarding the effectiveness of maskers when used in isolation.

\section{METHODS}

$T$ he study was a two-arm single-participant design of 18 adults with sensorineural tinnitus. Participants were included in the study after meeting the following criteria:

- adults (18 years + ) with tinnitus

- no history of mental problems or dementia

- severity of tinnitus $>30 \%$ on Tinnitus Primary Functions Questionnaire (TPFQ; Tyler et al, 2014)

- participants with hyperacusis were excluded

- participants using hearing aids were excluded

- participants with pulsatile or typewriter tinnitus, palatal myoclonus, and middle ear myoclonus were excluded.

Eighteen adults aged 46 to 72 years (mean age $=$ 60.1) were recruited from our database of tinnitus patients interested in participating in research. The study was carried out at the University of Iowa and Augustana College. Eight males and ten females participated. Duration of tinnitus varied from four months to 55 years (median $=10$ years; mean $=15.5$ years). The characteristics of the 18 participants are shown in Table 1.

Participants were informed about the purpose of this research and given a written informed consent before the onset of the trial. The study was approved by the Institutional Review Boards where the research was 
Table 1. Participant Characteristics and Duration of Their Tinnitus

\begin{tabular}{lcllc}
\hline Participant & Age & Gender & Location & $\begin{array}{c}\text { Duration of } \\
\text { Tinnitus (Years) }\end{array}$ \\
\hline 1 & 62 & Male & Augustana & 2 \\
2 & 65 & Female & lowa & 10 \\
3 & 58 & Male & Augustana & 10 \\
4 & 59 & Female & Augustana & 5 \\
5 & 58 & Female & lowa & 3 \\
6 & 46 & Male & lowa & 4 months \\
7 & 57 & Male & lowa & 30 \\
8 & 48 & Male & lowa & 20 \\
9 & 72 & Female & lowa & 15 \\
10 & 60 & Female & lowa & 3 \\
11 & 64 & Female & Augustana & 4 \\
12 & 58 & Male & Augustana & 2 \\
13 & 63 & Female & lowa & 5 \\
14 & 70 & Female & lowa & 55 \\
15 & 55 & Male & Augustana & 35 \\
16 & 65 & Female & Augustana & 30 \\
17 & 53 & Male & Augustana & 10 \\
18 & 68 & Female & Augustana & 25 \\
\hline & & & &
\end{tabular}

conducted. Participants were told that the study was intended to determine the effects of partial masking, and therefore, they would have two six-week trials, one with sound therapy and the other with no-sound therapy. They were randomly assigned the order of the treatment arms and we did an $\mathrm{AB}$ design with one half of the participants and a BA design with the other half of the participants.

In the first visit, participants listened to several sound therapy programs through the tinnitus maskers and selected up to three masking sounds to use during the at-home trial. They were encouraged to use the sound therapy as much as possible, including all waking hours. We acknowledged with them that this might not be possible or desirable for them. Alternatively, we encouraged them to wear the devices at times during the day when their tinnitus was most bothersome. Participants were encouraged to adjust the volume to a low and comfortable level to reduce the loudness and prominence of their tinnitus. We recommended that tinnitus maskers should be used to provide a partial masking, at a lower level to interfere less with speech (Tyler and Bentler, 1987). They were asked to rate their experience every week online for six weeks. After the six-week period, participants from both arms returned to the clinic and were crossed over to the other condition during the final six weeks of the trial. All participants were re-evaluated at the end of the trial.

A priori, we determined our analysis would focus on individuals rather than group data. Because individuals have different tinnitus percepts, reactions, and expectations, we did not expect to help all tinnitus patients (Tyler et al, 2006b; 2007b).
Participants were fit only with maskers (no hearing aids), and also no tinnitus counseling was provided during the study (handouts and counseling were offered at the end of the study; Tyler et al, 2008). For those who had hearing loss, we did not exclude these participants, provided that they could hear the masking sounds during the fitting of the devices. We also did not exclude based on the location of the tinnitus (unilateral, bilateral, or in the head).

There were four subjective outcome measures used in this study to evaluate the effectiveness of the masker: (a) tinnitus loudness, (b) tinnitus annoyance, (c) acceptability of the background sounds were rated using a numeric 0-100 interval scale, (d) and the 12-item TPFQ (Tyler et al, 2014; see Appendix) was administered before and after each condition.

Our research and clinical experience has led us to believe that tinnitus can affect patients in four primary functions, including (a) thoughts and emotions, (b) hearing, (c) sleep, and (d) concentration. The 12-item TPFQ was used in this study because it is a sensitive tool to determine treatment efficacy on the basis of only primary functions affected by tinnitus in the individual. Other questionnaires, such as the Tinnitus Functional Index (Meikle et al, 2012), includes questions related to secondary effects of tinnitus in the "enjoyment of life" and "relationships with family, friends, and other people." These secondary effects are just as likely to be influenced by a variety of daily life activities as they would be by a tinnitus treatment. Therefore, we believe the Tinnitus Functional Index is more likely to be influenced by other activities and, consequently, less sensitive to changes in tinnitus than a questionnaire focused on primary tinnitus handicaps. We also used the 12item TPFQ because it contains fewer numbers of questions and can be completed quickly. Therefore, the 12item TPFQ is a helpful scale to be used clinically and document treatment effects (Tyler et al, 2014).

Participants were asked to access an internet site one week before Visit 1 to complete preliminary tinnitus questionnaires to determine their eligibility for the study. Only those participants who had a score on the TPFQ of $>30 \%$ were included in the study. At Visit 1 , participants listened to several masker sounds through the tinnitus maskers and selected up to three sounds that reduced the prominence of their tinnitus and was acceptable to hear as a background sound. We fit the Signia Pure 7 Primax hearing aids with a tinnitus masker feature that was used for the at-home masker trial. The background sound therapy was generated from the Signia masker, using the same sampling rate as the amplified output $(12 \mathrm{kHz})$, and no mp3 files were used. The Signia devices were fitted bilaterally using open domes to allow environmental sounds to be heard. All participants were fit with receiver-in-canal (RIC) devices and standard receivers 
with an appropriately measured ear wire length. The devices were programmed using tinnitus therapy in the Connexx fitting software and the ConnexxLink.

For all participants, the tinnitus therapy was activated on the Signia device, and amplification was turned off. Participants were allowed to choose up to three therapeutic sounds to use in the tinnitus masker at-home trial. They were instructed to use a low level of the background sound, with the goal of reducing the prominence of their tinnitus. Therapeutic sounds (i.e., three static sounds-pink noise, white noise, and Brownian noise, and two modulated sounds-rocky beach and sandy beach) were played to each participant and participants rated on a one to five scale, their preference for the sound therapy in decreasing the prominence of their tinnitus. The top one, two, or three stimulus choices were then programmed into the tinnitus masker for the at-home trial. If none of the sounds were preferable, the remaining tinnitus therapy sound options were played to the participant to make a selection (i.e., two additional static sounds-white noise and speech noise, and two additional modulated soundspebble and boulder beach). Open dome fitting tips were connected to the maskers to enable the participants to listen to external sounds in the same manner as always. All fittings were completed using a standard receiver and an ear wire of appropriate length.

Participants were required to rate their tinnitus loudness and annoyance weekly using a secure, online portal. When participants were using the maskers, they were also requested to rate tinnitus sound therapy acceptability weekly. At the end of six weeks, all participants returned to the clinic and completed the tinnitus questionnaires. Those participants who started the trial without maskers were switched to using maskers and those who started with the maskers were switched to no masker as part of the crossover design. Again, all participants rated their tinnitus loudness and annoyance at the end of each week, for the next six weeks, using the online surveys. For those with maskers, they were also asked to rate the tinnitus sound therapy acceptability weekly.

At the conclusion of the study, the participants returned to the clinic for a final visit, at which time the devices were either returned or purchased at a discounted price. We did not provide additional compensation to the participants beyond the discount on the purchase of the masking devices. Amplification was activated at the end of the trial as needed for those participants who chose to purchase the maskers.

\section{RESULTS}

$\mathrm{T}$ hree participants dropped out from the study. Participant 5 was in the no-masker control condition for five weeks; tinnitus annoyance remained between
$70 \%$ and $100 \%$ and then she decided not to continue in the study. Participant 2 used the sound therapy for six weeks, did not report any benefit, and discontinued participation in the study. Participant 6 used the sound therapy for three weeks, found a $10 \%$ reduction in tinnitus annoyance, and then chose not to continue the study.

Figure 1 shows the TPFQ results for the total score for the no-sound and sound therapy condition. We consider a 13\% reduction in the TPFQ (Coelho et al, 2013; Tyler et al, 2014) to be significant. Five of 15 participants (33\%) showed a benefit on the overall TPFQ score. This suggests that the benefit of the maskers can have a positive effect on the perception and reactions to tinnitus. We have argued that questions on functions that are not affected by a symptom such as tinnitus render the questionnaire insensitive to treatment effects. For example, if a participant does not experience any difficulty sleeping, then questions related to sleeping will not show any benefit, even though the treatment might have been very effective for other functions. Therefore, we advocated that those questions be removed from the evaluation of a treatment (Tyler et al, 2006b; 2007b). For the derived score, we excluded those patients who presented with a score lower than $20 \%$ in each of the subscales, or total score, in the no-sound therapy condition. Eleven participants showed a score greater than $20 \%$ in the overall TPFQ before sound therapy, showing that these participants were bothered by their tinnitus in all four primary functions. Figure 2 shows the derived total TPFQ score for the 11 participants. There was a significant decrease in bothersome tinnitus with sound therapy, specifically in the overall TPFQ score for 5 of 11 participants (S11, S12, S15, S17, and S3). Most, although not all, patients benefited from

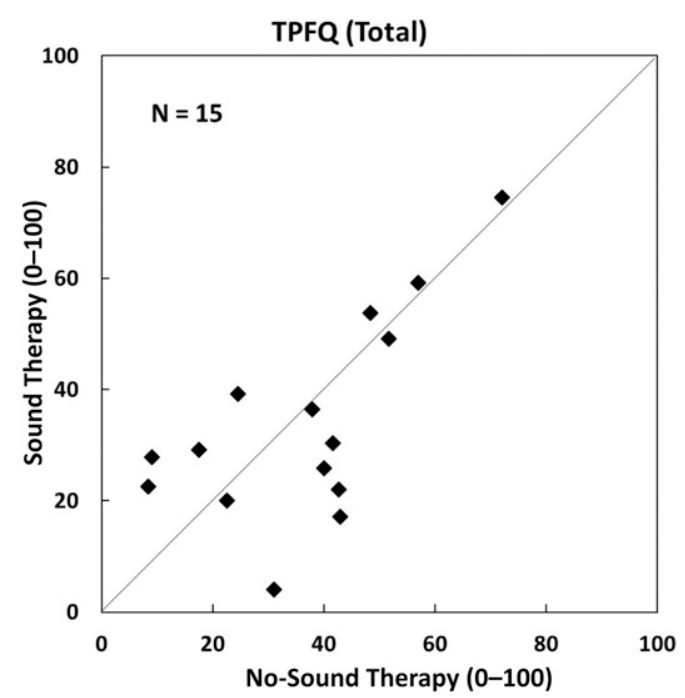

Figure 1. Individual total TPFQ scores for the no-sound ( $x$-axis) and sound therapy ( $y$-axis) conditions. 


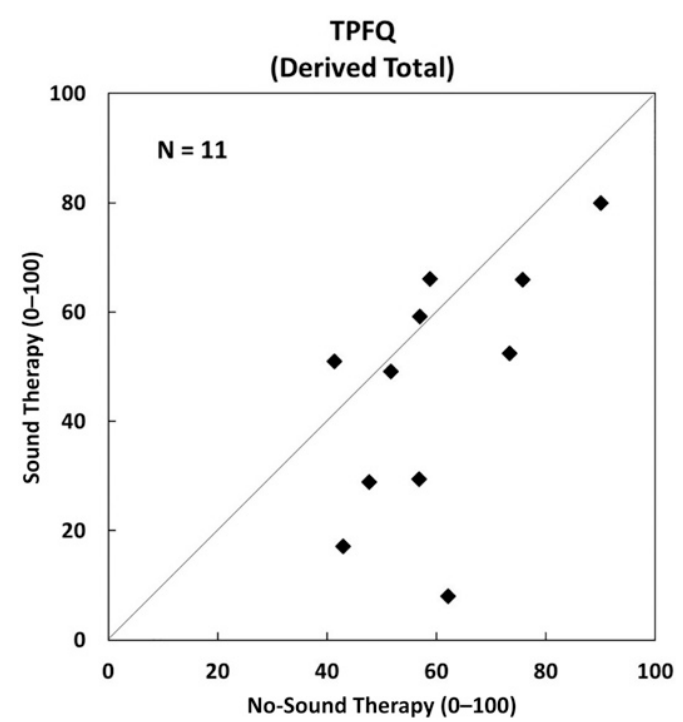

Figure 2. Derived total scores on TPFQ for the no-sound ( $x$-axis) and sound therapy ( $y$-axis) conditions.

the sound therapy to some degree. For example, S3, who had a significant reduction in his TPFQ score with tinnitus masker use, wore the tinnitus maskers for 12 hours per day and reported that they reduced his tinnitus loudness most days. He also reported conversation was easier with the tinnitus maskers. S3 had chronic bilateral tinnitus for over 10 years because of aging and medications. The tinnitus maskers also provided some residual inhibition for up to $30-45$ minutes at night after he removed the devices. The post-masking reduction improved his sleep.

Figure 3 shows the TPFQ-derived scores from the four subscales, thoughts and emotions, hearing, sleep, and concentration for the no-sound ( $x$-axis) and sound therapy ( $y$-axis) conditions. In thoughts and emotions, there were only four participants who reported a significant effect of tinnitus on their thoughts and emotions before sound therapy. This highlights the observation that this group was not very distressed by their tinnitus. We observed that, of those participants who were bothered by their tinnitus emotionally, three of four participants (S11, S12, and S16) showed a benefit on emotion-derived scores with sound therapy. S16 had improved scores on the thoughts and emotions subscale with the use of the tinnitus maskers, although no additional improvements in other areas (sleep, hearing, etc.). This participant had bilateral, bothersome tinnitus for over 30 years and reported that the tinnitus maskers provided a distraction from her tinnitus, but she still heard her tinnitus with the maskers even when she increased the level of the sound therapy. If she increased the level of the background sounds, then her speech understanding and TV listening were negatively affected. Thus, there was no further benefit observed on the hearing subscale of the TPFQ. Finally, she reported that at night, if she thought about the masking sound, she would calm down as evidenced by improvements in the thoughts and emotions scores.

The results from the hearing subscale indicated that eight participants were very bothered by their hearing ability. We have noted that it is difficult for some participants to distinguish the hearing difficulties caused by the hearing loss from that caused by the tinnitus (Tyler and Baker, 1983; Henry et al, 2015). Three of eight participants (S11, S15, and S3) showed a benefit on hearing-derived scores with the use of the tinnitus maskers. To highlight improvements from S15, he reported at the end of the trial that his tinnitus was not as noticeable during the day and that the tinnitus masking sound was helpful; and acceptable to have in the background, consistent with his improvement on the hearing subscale of the TPFQ. This participant used the tinnitus maskers for nearly eight hours per day during the at-home trial and preferred the modulated ocean waves sound. This participant had bilateral tinnitus for over 30 years and normal sloping to mild sensorineural hearing loss.

Regarding sleep, nine participants reported a sleep disturbance because of their tinnitus before using tinnitus maskers, and five of the nine participants (S11, S14, S15, S3, and S9) showed a benefit of the sound therapy based on the sleep-derived score. S9 and S14 reported improvements in sleep and not in other areas impacted by tinnitus. Specifically, S9 had bilateral tinnitus for 15 years and reported that nothing made her tinnitus worse, or better. She reported that the tinnitus maskers were initially very acceptable in reducing her tinnitus, although this rating dropped during the athome trial, suggesting that the benefit was limited to improving her sleep only.

Concentration was also reportedly affected by tinnitus for 8 of the 15 participants before sound therapy. Of those affected, half of the participants (four participants: S11, S12, S15, and S17) reported an improvement in their concentration with the sound therapy. S12 found significant benefit from the tinnitus maskers in several areas impacted by tinnitus, including his thoughts and emotions and concentration abilities. He reported tinnitus for two years and had sought help for his tinnitus from audiologists and ENT specialists. He was very motivated to find an effective treatment for tinnitus, and wore the tinnitus maskers about two to three days per week because of his busy work schedule. On some days, his tinnitus reacted negatively to the tinnitus maskers after wearing them; however, he did report that tinnitus was reduced consistently with his improvement in scores on the TPFQ.

We believe it is insightful to view some diverse, individual differences highlighted throughout the masker at-home trial on tinnitus annoyance. Figure 4A shows a high tinnitus annoyance rating for S3 for the first five 
TPFQ

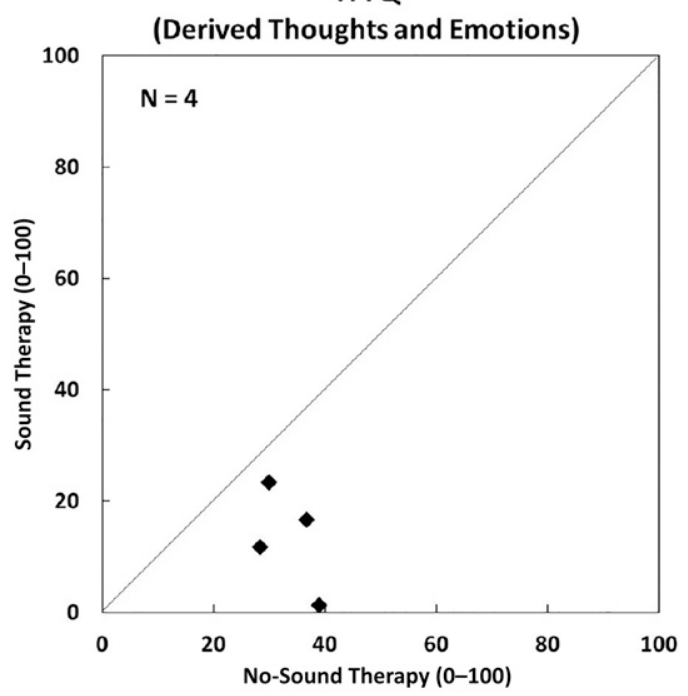

TPFQ

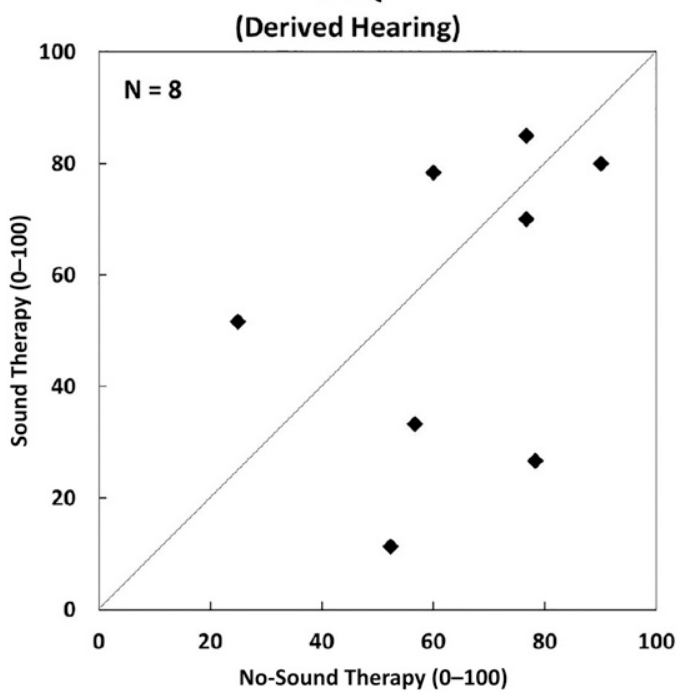

TPFQ

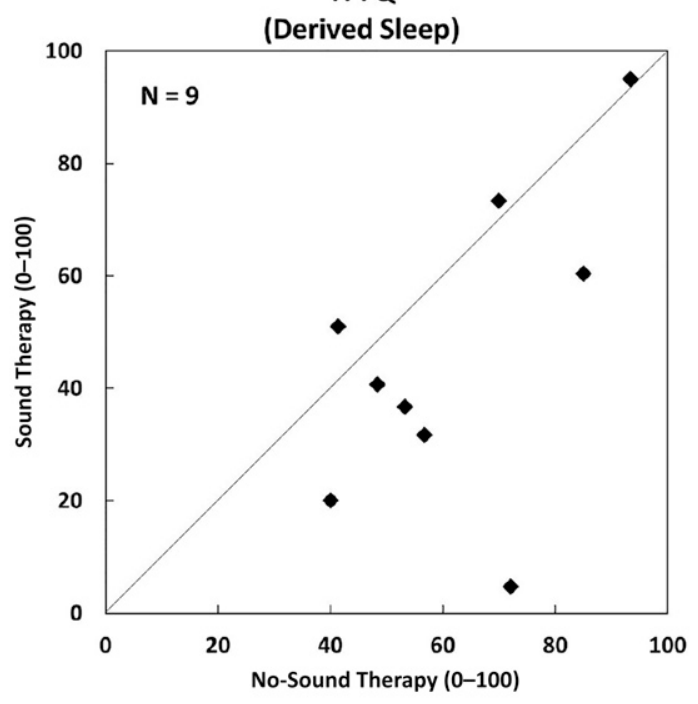

TPFQ

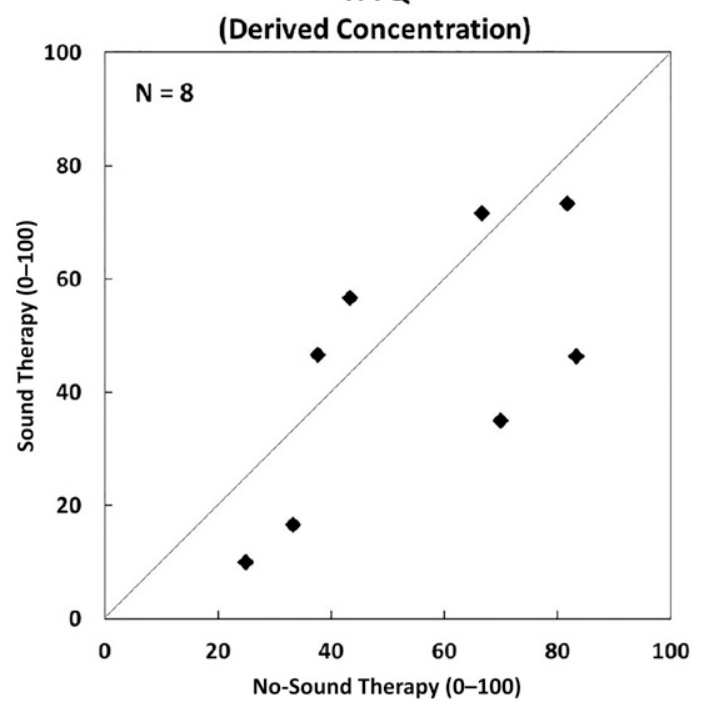

Figure 3. Tinnitus Primary Function Questionnaire-derived scores from thoughts and emotions, sleep, hearing, and concentration subscale for the no-sound ( $x$-axis) and sound therapy ( $y$-axis) conditions.

weeks of using the sound therapy. After five weeks, the patient apparently began to find that the sound therapy was helpful and annoyance ratings decreased from $65 \%$ to $18 \%$ on visit 7 . This benefit continued even after the sound therapy was removed. It may be that the participant knew that there was a successful treatment for him; he was no longer helpless and there was something he could do for his tinnitus.

Figure $4 \mathrm{~B}$ shows annoyance ratings for $\mathrm{S} 8$. There was a drop from $45 \%$ to $30 \%$ in annoyance when this participant initially switched from no-sound therapy to sound therapy. However, annoyance increased substantially over the next several weeks using the sound therapy, perhaps because the participant realized that the tinnitus was not going to go away.
This participant reported constant bilateral tonal tinnitus caused by noise exposure, with normal lowfrequency hearing sloping to a bilateral, moderately severe sensorineural hearing loss. He did not have loudness hyperacusis that would explain the increased tinnitus annoyance with use of the masker; however, he stated that "nothing makes his tinnitus better," and his acceptability of the masker decreased overtime, as he realized the tinnitus percept continued. Figure 4C shows annoyance ratings for S11. Annoyance ratings were between $50 \%$ and $80 \%$ without the sound therapy. When he switched over to sound therapy, no change was observed during the first two weeks. Interestingly, after that time, annoyance dropped steadily. Participant S11 reported that her bilateral tinnitus 

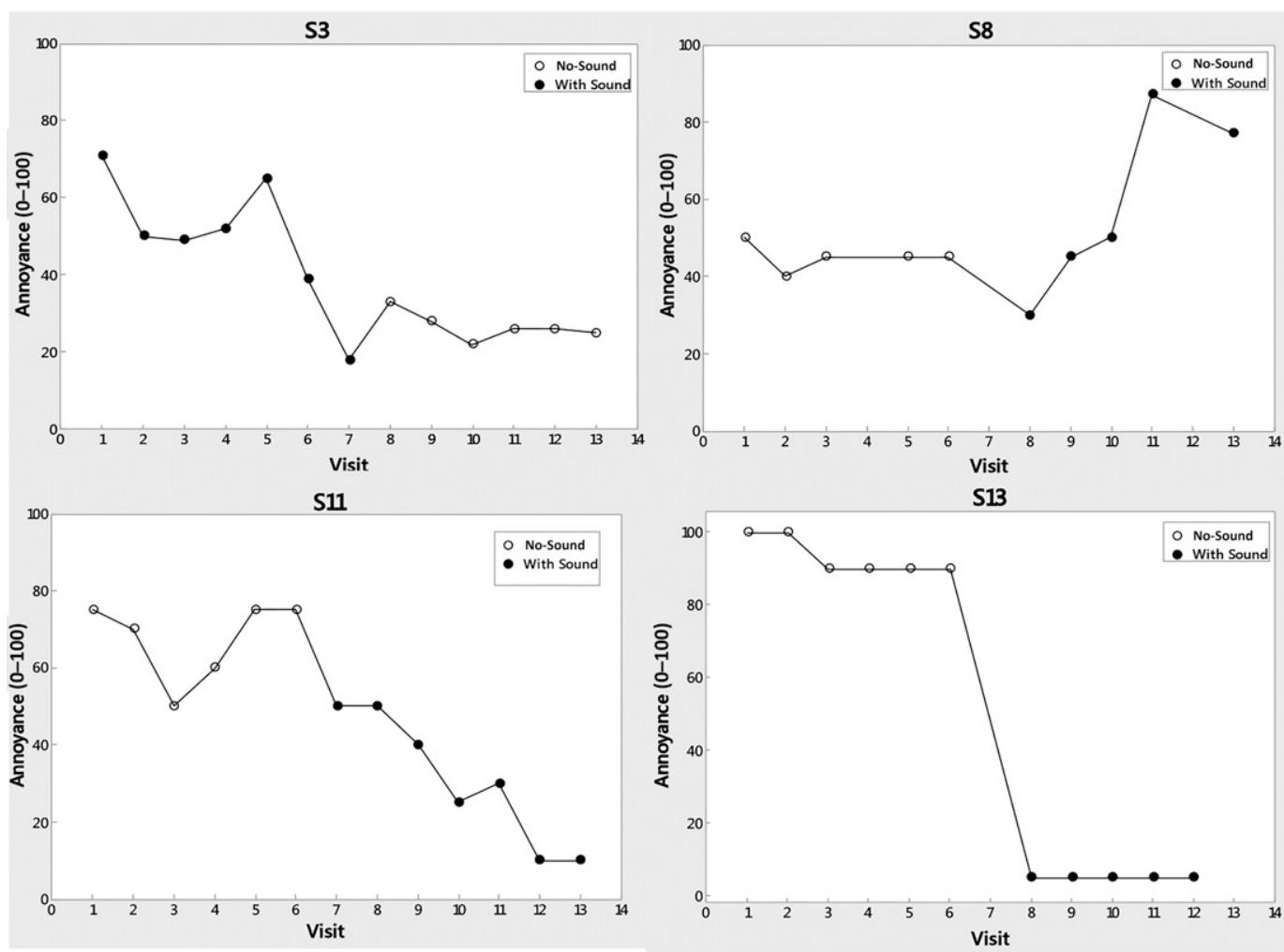

Figure 4. Individual differences on tinnitus annoyance ratings at the no-sound and sound therapy conditions.

was caused by noise exposure and had a slight sloping to a moderately severe, high-frequency sensorineural hearing loss. She used the tinnitus maskers for over eight hours a day during the at-home trial and reported significant improvements on the TPFQ. Consistent with this drop in tinnitus annoyance, S11 stated that it was a great experience to use the maskers and indicated that her tinnitus felt "thinner, not as noticeable."

Figure 4D shows annoyance ratings for S13, which illustrate the potential for dramatic tinnitus relief using sound therapy.

There were some interesting observations made regarding the relationship between tinnitus loudness, tinnitus annoyance, and the sound therapy acceptability. To investigate these relationships, we created scatterplots based on data for each participant and averaged the last three subjective ratings during the at-home trial using the sound therapy condition. For three participants, 7,8 , and 13 , only one or two ratings were available, and these data were used to calculate their average ratings for tinnitus loudness and annoyance. In Figure 5, the ratings of tinnitus loudness and annoyance were compared. Generally, for an individual, a louder tinnitus is considered more annoying, so that as tinnitus loudness ratings increase, there is a higher rating for tinnitus annoyance.

However, in Figure 6, the relationship between annoyance and loudness was not clearly related to the ac- ceptability of the sound therapy across individuals. In some, the tinnitus might decrease in loudness, but if their hope was that the tinnitus would go away, they might not be satisfied. In others, the loudness of the tinnitus might not change, but the background sound might make it easier to experience and thus decreasing its annoyance.

\section{DISCUSSION}

$\mathrm{T}$ his controlled study provides evidence that many tinnitus patients benefit from sound therapy. Tinnitus patients may suffer from problems in concentration, hearing, sleep, and thoughts and emotions (Stouffer and Tyler, 1990). The TPFQ was used to evaluate how tinnitus affects these four primary areas of a patient's life (Tyler et al, 2014), and we noted that a large percentage of patients experience sleep disturbance because of their tinnitus similar to previous studies (Tyler and Baker, 1983; McKenna, 2000; McKenna and Daniel, 2006). In the present study, the benefit of maskers was observed especially in the areas of sleep (five of nine), thoughts and emotions (three of four), and concentration (four of eight). We also noted that TPFQ and annoyance data complemented each other well.

Although not all patients benefited, clearly some showed a relief in tinnitus annoyance with the use of 


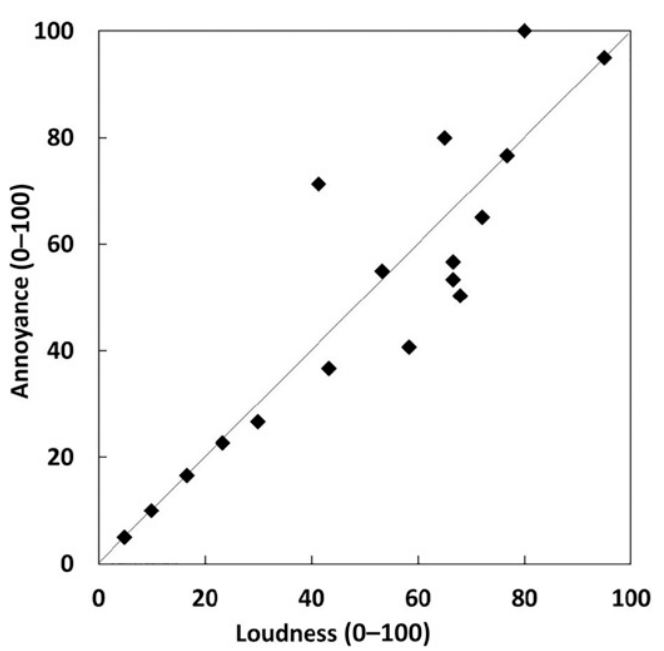

Figure 5. Individual ratings for tinnitus loudness ( $x$-axis) and annoyance ( $y$-axis).

maskers alone. Dauman and Tyler (1992) proposed that the overall impact of tinnitus on a patient is influenced by the characteristics not only of tinnitus but also of that particular patient. Therefore, we believe it is critical to examine individual results to understand treatment effects in patients with bothersome tinnitus because not all tinnitus patients are likely to benefit identically to the same treatment. In this study, we identified 5 of 11 participants who benefited from sound therapy based on the derived TPFQ score. We wish to emphasize that the American Academy of Otolaryngology-Head and Neck Surgery Clinical Practice Guidelines (Tunkel et al, 2014) also cautioned about looking at "mean data" in sound therapy trials because of individual difference and different tinnitus subtypes. They also noted "roadblocks" to some individuals receiving sound therapy, including that the use of background sound (including participating in a sound therapy trial) can create an elevated "arousal state" to the external the sound therapy. They noted that "patients seeking sound therapy must be provided realistic expectations" and concluded that "sound therapy may be a reasonable management option to offer patients when appropriate counseling is provided by the clinician." We agree.

Large individual differences were seen in benefit with the use of sound therapy. For example, Figure $4 \mathrm{C}$ shows one participant who shows a steady drop in annoyance, whereas Figure $4 \mathrm{D}$ shows another with a large and rapid advantage in annoyance with the use of maskers. The improvement on tinnitus annoyance following the use of maskers indicates that this is indeed useful, and some patients obtain immediate relief from their tinnitus with maskers (Folmer et al, 2006). For other patients, annoyance ratings were higher when using sound therapy (as shown in Figures 4A and B). This raises an important issue that not all patients benefit from maskers. It is important to appreciate that sound therapy provides a background sound that might be unpleasant for some patients, might interfere with speech perception and localization for others, and does not consistently make the tinnitus go away for all patients.

These individual data accurately show the variability that is typical when evaluating interventions in tinnitus patients. Our research and clinical experience has led us to consider not only the tinnitus as an isolated problem, but we address all the patient's difficulties (Tyler and Babin, 1986; see also; Mohr and Hedelund, 2006). Tinnitus Activities Treatment (Tyler et al, 2006a; 2007a) focuses on the four primary functions affected by tinnitus (thoughts and emotions, concentration, hearing, and sleep) and often includes sound therapy focused on partial masking with music or noise setting a low level to provide relief from tinnitus (Tyler et al, 2007a).
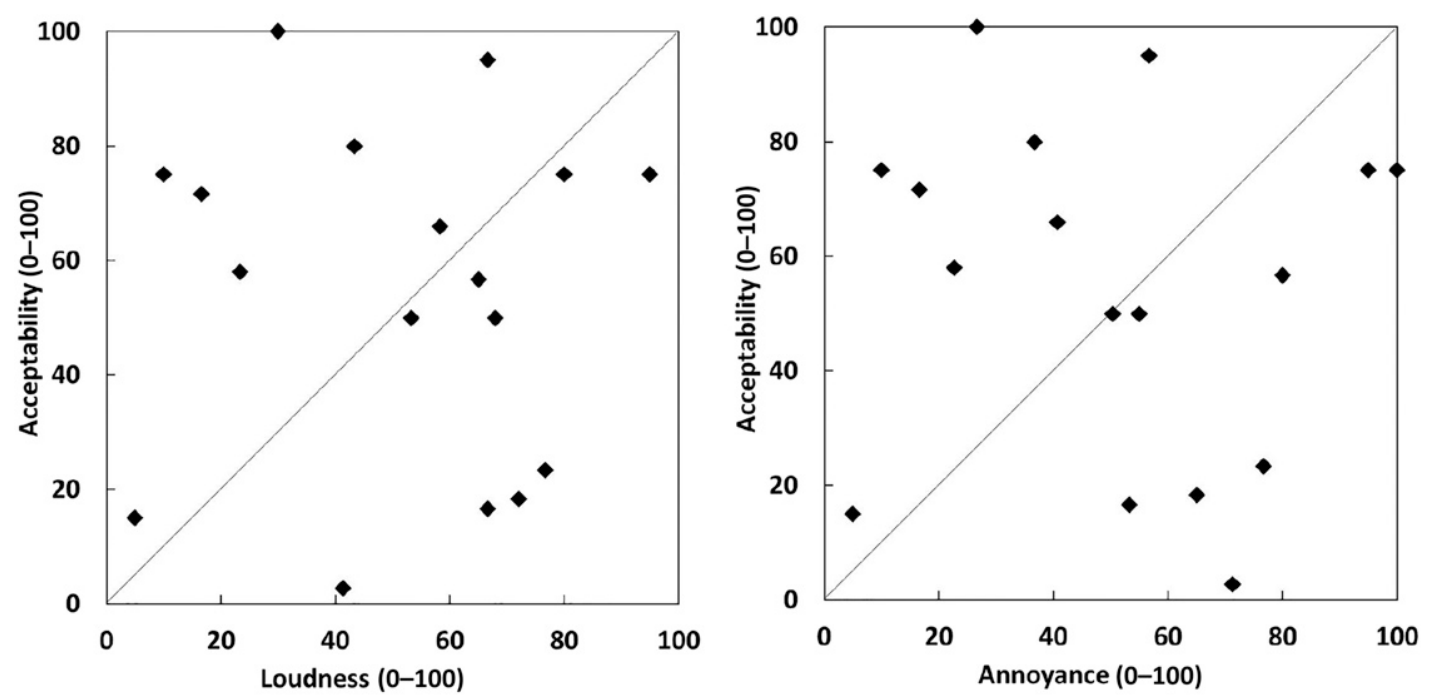

Figure 6. Individual ratings for tinnitus loudness (left) and annoyance (right) and acceptability of sound therapy. 
In this study, we examined the efficacy of using tinnitus maskers alone. As such, the protocol we followed did not provide any other intervention to improve symptoms for patients with bothersome tinnitus. Within a clinical setting, it is important to consider many aspects that were not implemented in this study. For example, in this experiment, it did not matter whether the patient's hearing loss or tinnitus was unilateral or bilateral. If taken into consideration, some participants would have benefited from hearing aid amplification, but they were not given amplification. Others might have been helped with counseling, but that was not provided in this study. Likely, more participants would have shown a benefit with sound therapy if hearing aids and counseling had also been provided. Patients with persistent, bothersome tinnitus can be helped by many treatment options, especially by counseling, hearing aids, and sound therapy (Tunkel et al, 2014). However, it is important to determine which are the most helpful and work best for each patient (Tyler, 2006). It is crucial for clinicians to understand that each tinnitus patient is unique and customized treatment may be required (Tyler et al, 2006a; 2007a).

It is appropriate to comment on the benefits of maskers to patients with sleep difficulties. When participants used the masker during the day, tinnitus was less bothersome and they felt more relaxed and more "in control" and slept better. In addition, some experience relief from tinnitus after turning the masker off at bedtime, thus making it easier to fall asleep.

It is important to draw a cautionary note about the participants in this experiment. It is possible that a "placebo affect" occurred, that is, some might have felt that just receiving any attention for their tinnitus, in this case sound therapy, would help them no matter what. We attempted to limit this by being clear with the participants at the onset; the study was aimed at trying to determine if sound therapy did, or did not, help. In this study, participants were recruited from a website and some either wanted a magic pill or were willing to try anything to reduce the prominence of their tinnitus (Tyler, 2012). Thus, this studied sample is not the same as a typical clinic population. Some patients come into an audiology clinic wanting to try maskers, whereas others do not, but they are provided with a trial period to realize the benefit of the masker.

In conclusion, this study demonstrates that many patients with tinnitus benefit from sound therapy alone, using partial masking (not total masking or mixingpoint masking). Thirty-three percent of our participants with bothersome tinnitus benefited from maskers without any counseling whatsoever. Within that responsive group, 38-75\% of them experienced improved quality of life in the domains of thoughts and emotions, sleep, concentration, and hearing. Maskers remain as clinically useful devices for many patients seeking relief from bothersome tinnitus. It is noteworthy that the American Academy of Otolaryngology-Head and Neck Surgery Clinical Practice Guidelines (Tunkel et al, 2014) did note that "although not for everyone, these devices can be very helpful for many." They also noted that "sound therapy may be a reasonable management option to offer patients when appropriate counseling is provided by the clinician."

\section{REFERENCES}

Bauer CA, Berry JL, Brozoski TJ. (2017) The effect of tinnitus retraining therapy on chronic tinnitus: a controlled trial. Laryngoscope Investig Otolaryngol 28:166-177.

Byiers B, Reichle J, Symons FJ. (2012) Single subject experimental design for evidence-based practice. Am J Speech Lang Pathol 21:397-414.

Coelho C, Witt S, Ji H, Hansen M, Gantz B, Tyler R. (2013) Zinc to treat tinnitus in the elderly: a randomized placebo controlled crossover trial. Otol Neurotol 34:1146-1154.

Coles RRA. (1987) Tinnitus and its management. In: Stephens SDG, Kerr AG, eds. Scott-Brown's Otolaryngology. Guildford, UK: Butterworth, 368-414.

Dauman R, Tyler RS. (1992) Some considerations on the classification of tinnitus. In: Aran J-M, Dauman R, eds. Tinnitus 91-Proceedings of the Fourth International Tinnitus Seminar. Bordeaux, France: Kugler Publications, 225-229.

Folmer RL, Martin WH, Shi Y, Edlefsen LL. (2006) Tinnitus sound therapies. In: Tyler RS, ed. Tinnitus Treatment: Clinical protocols. New York, NY: Thieme, 176-186.

Hazell JWP, Wood SM, Cooper H, Stephens SDG, Corcoran AL, Coles RRA, Baskill JL, Sheldrake JB. (1985) A clinical study of tinnitus maskers. $\mathrm{Br}$ J Audiol 19:65-116.

Henry J, Griest S, Zaugg TL, Thielman E, Kaelin C, Galvez G, Carlson KF. (2015) Tinnitus and hearing survey: a screening tool to differentiate bothersome tinnitus from hearing difficulties. Am J Audiol 24(1):66-77.

Henry J, McMillan G, Dann S, Bennett K, Griest S, Theodoroff S, Silverman SP, Whichard S, Saunders G. (2017) Tinnitus management: randomized controlled trial comparing extended-wear hearing aids, conventional hearing aids, and combination instruments. J Am Acad Audiol 28:546-561.

Henry J, Wilson P. (2001) The Psychological Management of Chronic Tinnitus: A Cognitive-Behavioral Approach. Boston, MA: Allyn \& Bacon.

Henry JA, Zaugg TL, Schechter MA. (2005) Clinical guide for audiologic tinnitus management II: Treatment. Am J Audiol 14:49-70.

Hobson J, Chisholm E, El Refaie A. (2010) Sound therapy (masking) in the management of tinnitus in adults. Cochrane Database Syst Rev 12(11):1-26. CD006371.

Jastreboff PJ. (2000) Tinnitus habituation therapy (THT) and tinnitus retraining therapy (TRT). In: Tyler RS, ed. Tinnitus Handbook. San Diego, CA: Singular, 357-376.

Jastreboff PJ, Hazell JW. (1993) A neurophysiological approach to tinnitus: clinical implications. Br J Audiol 27(1):7-17.

Kochkin S, Tyler R, Born J. (2011) MarkeTrak VIII: prevalence of tinnitus and efficacy of treatments. Hear Rev 18(12):10-26. 
McKenna L. (2000) Tinnitus and insomnia. In: Tyler RS, ed. Tinnitus Handbook. San Diego, CA: Singular, 59-84.

McKenna L, Daniel HC. (2006) Tinnitus-related insomnia treatment. In: Tyler RS, ed. Tinnitus Treatment: Clinical Protocols. New York, NY: Thieme, 81-95.

Meikle MB, Henry JA, Griest SE, Stewart BJ, Abrams HB, McArdle R, Myers PJ, Newman CW, Sandridge S, Turk DC, Folmer RL, Frederick EJ, House JW, Jacobson GP, Kinney SE, Martin WH, Nagler SM, Reich GE, Searchfield G, Sweetow R, Vernon JA. (2012) The tinnitus functional index: development of a new clinical measure for chronic, intrusive tinnitus. Ear Hear 33:153-176.

Moffat G, Adjout K, Gallego S, Thai-Van H, Collet L, Norena AJ. (2009) Effects of hearing aid fitting on the perceptual characteristics of tinnitus. Hear Res 254:82-91.

Mohr AM, Hedelund U. (2006) Tinnitus person-centered therapy. In: Tyler RS, ed. Tinnitus Treatment: Clinical Protocols. New York, NY: Thieme, 198-216.

Sandlin RE, Olsson RJ. (1999) Evaluation and selection of maskers and other devices used in the treatment of tinnitus and hyperacusis. Trends Amplif 4(1):6-26.

Schaette R, Konig O, Hornig D, Gross M, Kempter R. (2010) Acoustic stimulation treatments against tinnitus could be most effective when tinnitus pitch is within the stimulated frequency range. Hear Res 269:95-101.

Stouffer JL, Tyler RS. (1990) Subjective tinnitus loudness. Hear Instrum 41(2):17-19.

Surr RK, Montgomery AA, Mueller HG. (1985) Effect of amplification on tinnitus among new hearing aid users. Ear Hear 6(2): 71-75.

Sweetow R. (2000) Cognitive behavior therapy for tinnitus. In: Tyler RS, ed. Tinnitus Handbook. San Diego, CA: Singular Publishing Group, 297-311.

Tunkel DE, Bauer CA, Sun GH, Rosenfeld RM, Chandrasekhar SS, Cunningham ER, Jr., Archer SM, Blakley BW, Carter JM, Granieri EC, Henry JA, Hollingsworth D, Khan FA, Mitchell S, Monfared A, Newman CW, Omole FS, Phillips CD, Robinson SK, Taw MB, Tyler RS, Waguespack R, Whamond EJ. (2014) Clinical practice guideline: tinnitus. Otolaryngol Head Neck Surg 151(2, Suppl):S1-S40.
Tyler RS. (2006) Neurophysiological models, psychological models, and treatments for tinnitus. In: Tyler RS, ed. Tinnitus Treatment: Clinical Protocols. New York, NY: Thieme, 1-22.

Tyler RS. (2012) Patient preferences and willingness to pay for tinnitus treatment. J Am Acad Audiol 23:115-125.

Tyler RS, Babin RW. (1986) Tinnitus. In: Cummings CW, Fredrickson JM, Harker L, Krause CJ, Schuller DE, eds. Otolaryngology -Head and Neck Surgery. St. Louis, MI: C.V. Mosby Co, 3201-3217.

Tyler RS, Baker LJ. (1983) Difficulties experienced by tinnitus sufferers. J Speech Hear Disord 48(2):150-154.

Tyler RS, Bentler RA. (1987) Tinnitus maskers and hearing aids for tinnitus. Semin Hear 8(1):49-61.

Tyler RS, Gehringer AK, Noble W, Dunn CC, Witt SA, Bardia A. (2006a) Tinnitus activities treatment. In: Tyler RS, ed. Tinnitus Treatment: Clinical Protocols. New York, NY: Thieme, $116-132$.

Tyler RS, Gogel SA, Gehringer AK. (2007a) Tinnitus activities treatment. Prog Brain Res 166:425-434.

Tyler RS, Haskell G, Gogel S, Gehringer A. (2008) Establishing a tinnitus clinic in your practice. Am J Audiol 17:25-37.

Tyler RS, Ji H, Perreau A, Witt S, Noble W, Coelho C. (2014) Development and validation of the Tinnitus Primary Function Questionnaire. Am J Audiol 23:260-272.

Tyler RS, Noble WG, Coelho C. (2006b) Considerations for the design of clinical trials for tinnitus. Acta Otolaryngol 126:44-49.

Tyler RS, Oleson J, Noble W, Coelho C, Ji H. (2007b) Clinical trials for tinnitus: study populations, designs, measurement variables, and data analysis. Prog Brain Res 166:499-509.

Vernon JA. (1977) Attempts to relieve tinnitus. J Am Audiol Soc 2: 124-131.

Vernon JA, Meikle MB. (2000) Tinnitus masking. In: Tyler RS, ed. Tinnitus Handbook. San Diego, CA: Singular Publishing Group, 313-355.

Vernon JA, Schleuning A. (1978) Tinnitus: a new management. Laryngoscope 88:413-419. 


\section{APPENDIX}

Tinnitus Primary Function Questionnaire (12-item version)

Please indicate your agreement with each statement on a scale from 0 (completely disagree) to 100 (completely agree).

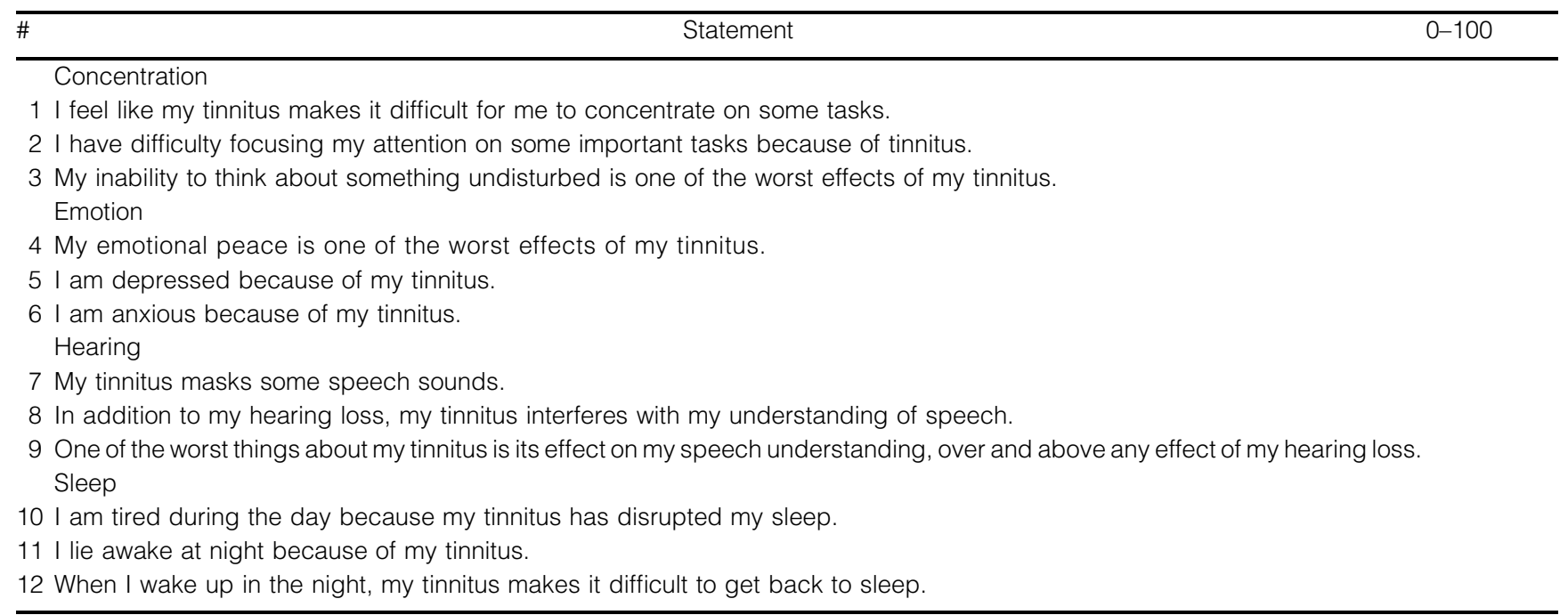

